\title{
Relación de la inteligencia ejecutiva y la praxia constructiva en niños del primer al tercer grado de primaria en dos colegios de gestión privada en la ciudad de Lima y Callao
}

Relationship of the executive intelligence and constructive praxia in children of the first to third grade in two private schools of management in the city of Lima and Callao

\author{
José Carlos Rivera B. ${ }^{1}$, César Sarria J., Pedro Garcia P. \\ Universidad Nacional Mayor de San Marcos, Lima, Perú
}

(RECIBIDO 28/09/2015, AcEPTADo 02/03/2016)

\begin{abstract}
RESUMEN
El objetivo del estudio fue buscar evidencia de la relación entre la inteligencia ejecutiva y la praxia constructiva (funciones cognitivas del pensamiento espacial), en el marco de la psicogenética piagetana (Piaget, 1972; Piaget et Inhelder, 1972) y la psiconeurología de A. Luria (1974, 1977), mediante la Escala Ejecutiva Borelli-Oléron (Borelli y Oléron, 2000) y el Test de Praxia Constructiva Tridimensional (Benton, 2010).

Se evaluó a 54 escolares del primer al tercer grado de primaria, de ambos sexos, provenientes de dos colegios de la ciudad de Lima y Callao, cuyo promedio de edad fue 7.60 (DE = 0.89). Las puntuaciones totales de la escala Borelli-Oléron (B-O) en la Muestra 1 variaron entre 33.00 y 61.50 , mientras que las puntuaciones totales de la escala Benton Tridimensional (BTd) en la Muestra 2 estuvieron entre 5.00 y 28.00.

El contraste por edad operatoria comparó la puntuación total obtenida en las escalas por los sujetos en los grupos concreto y preconcreto en cada muestra, donde los resultados en la escala B-O $(t=2.54 ; g l=10 ; p=0.03)$ y en la escala BTd $(t=2.71 ; g l=10 ; p=0.02)$ indicaron que las escalas discriminan a los sujetos por edad operatoria.

La hipótesis nula acerca de la diferencia de las puntuaciones de las escalas en cada muestra (según grado escolar, sexo y nivel de rendimiento académico) equiparó las muestras evaluadas (con tan solo una de las escalas) y permitió un matching o 'emparejamiento' (muestras equivalentes) para la prueba de asociación de las escalas donde la correlación fue positiva y muy significativa $(r=0.60 ; p=0.001)$.

Por lo tanto, se consiguió evidencia de la relación entre inteligencia ejecutiva y praxia constructiva. También se probó la capacidad de discriminación genética por edad operatoria en ambas escalas.
\end{abstract}

Palabras clave: Inteligencia ejecutiva, praxia constructiva, funciones cognitivas del pensamiento espacial, discriminación genética por edad operatoria: concreto y preconcreto, matching.

1 Profesor asociado de la Facultad de Psicología de la UNMSM. E-mail: jocarivera@gmail.com 


\begin{abstract}
The objective of this study was to find evidence of a relationship between the executive intelligence and the constructive praxia (cognitive functions of the spatial thinking), in the framework of the psicogenetic piagetana (Piaget, 1972; Piaget et Inhelder, 1972) and the psiconeurology of A. Luria (1974, 1977), using the Scale Executive Borelli-Oleron (Borelli and Oléron, 2000) and the test of Three Dimensional Constructive Praxia (Benton, 2010).

We evaluated 54 schoolchildren from the first to the third grade of both sexes, from two schools in the city of Lima and Callao whose average age was $7.60(\mathrm{SD}=0.89)$. Total scores for the scale Borelli-Oleron (B-O) in the sample 1 varied between 33.00 and 61.50 while the total scores of the scale Benton Three Dimensional (BTD) in sample 2 were between 5.00 and 28.00 .

By contrast the operative age compared the total score on the scales by the subjects in the concrete and preconcrete groups in each sample where the results in the scale B-O $(t=$ 2.54; $d f=10 ; p=0.03)$ and BTD scale $(t=2.71 ; d f=10 ; p=0.02)$ indicated that the scales discriminate subjects by operative age.

The null hypothesis about the difference in the scores of the scales in each sample (by grade level, gender and level of academic performance) equated the evaluated samples (with only one of the scales) and allowed a matching (equivalent samples) for the association test scales where the correlation was positive and highly significant $(r=0.60 ; p=0.001)$.

Therefore, there was evidence of the relationship between executive intelligence and constructive praxia. Also tested the ability of genetic discrimination by operative age in both scales.
\end{abstract}

Keywords: Executive Intelligence, constructive praxia, cognitive functions of the spatial thinking, genetic discrimination by operative age: concrete and preconcrete, matching.

\title{
INTRODUCCIÓN
}

El objetivo del estudio es relacionar la inteligencia ejecutiva y la praxia constructiva como funciones cognitivas del dominio de la representación espacial en una población escolar del primer al tercer grado de primaria, según sexo y nivel de rendimiento académico.

La interrogante que se busca responder en el estudio es si procede establecer esa relación mediante dos pruebas: una de carácter neuropsicológico, el Test de Praxia Constructiva Tridimensional (Benton, 2010), y otra de carácter psicogenético, la Escala Ejecutiva Borelli-Oléron (2000). Ambas pruebas poseen en común que la tarea que realiza el sujeto examinado consiste en establecer relaciones espaciales entre diversos objetos a partir de la presentación de modelos bidimensionales que el sujeto traduce a formas tridimensionales. Las pruebas implican funciones cognitivas que se enmarcan en el dominio del comportamiento espacial donde están comprometidos, en una, los mecanismos sensomotores de la corteza cerebral y, en la otra, el desarrollo psicogenético de la noción de espacio.

La cognición, en el campo del comportamiento espacial, se basa en procesos neuropsicológicos y psicogenéticos que se van formando desde el nacimiento como procesos sensoriomotores hasta los 2 años de edad, para luego iniciarse 
en formas representacionales del espacio, como se observa en las pruebas de la Escala Ejecutiva Borelli-Oléron (escala B-O) y los modelos del Test de Praxia Constructiva Tridimensional de Benton o escala Benton Tridimensional para abreviar (escala BTd).

Gracias a la Praxia Constructiva (PC) y la Inteligencia Ejecutiva (IE), el niño puede adquirir aprendizajes sensoriomotores complejos: dibujar modelos, orientar objetos en el espacio, etc., y, por tanto, son básicos para procesar la información perceptual en la educación escolar y en el manejo del medio físico. La escala ejecutiva establece un patrón de discriminación genética para edades de 4 a 9 años y la escala de praxia constructiva detecta las dispraxias como las apraxias, las primeras de carácter evolutivo, de 6 a 12 años, y las segundas por lesiones del sistema sensomotor de la corteza cerebral de los adultos.

En un trabajo anterior con la escala B-O, postulamos, según Piaget et Inhelder en 1966, que las fuentes de la imagen mental reproductora “(...) están íntimamente relacionadas a los comportamientos práxicos los que a su vez constituyen las bases de la inteligencia ejecutiva (...)" (Rivera, 2011: 40).

Consideramos que la escala BTd se enmarca perfectamente en los hallazgos de Luria desde mediados del siglo pasado, como veremos más adelante. Relacionar la IE y la PC, más allá de la evidencia empírica, es un intento práctico por integrar la psicogenética piagetana con la psiconeurología de Luria, en lo que vendría a ser una neuropsicogenética.

Teóricamente, el presente estudio se enmarca dentro de la orientación psiconeurológica, siguiendo el modelo de unidades funcionales propuesto por Alexander Romanovich Luria, quien coincide con la teoría psicogenética de Jean Piaget, al decir que “(...) las funciones psíquicas superiores se forman en el proceso de ontogénesis, pasando en este tiempo una serie de estadios sucesivos (...) ideas que fueron expuestas en las investigaciones de Piaget $(1947,1955)$ " (Luria, 1977: 39).

Al reunir dos teorías provenientes de la neuropsicología de Luria y de la psicogenética piagetana mediante instrumentos propios, estamos dando un paso hacia la integración de ambos enfoques para aportar a las neurociencias cognitivas en el ámbito del desarrollo intelectual de las funciones cognitivas del pensamiento espacial; en el terreno de la práctica profesional tendríamos una batería, a ampliarse posteriormente, para el examen clínico diferencial de la evolución de las funciones ejecutivas, lo que nos permitiría dar pautas para la rehabilitación neuropsicológica a la población escolar infantil.

En consecuencia, planteamos como hipótesis del estudio comprobar si la praxia constructiva y la inteligencia ejecutiva están relacionadas en virtud de pertenecer al comportamiento espacial, observando sus resultados en niños del primer al tercer grado de educación básica, en relación con la edad operatoria concreta y preconcreta, sexo y rendimiento escolar (bajo, medio y alto).

Los antecedentes conocidos se remontan a la década del 70, cuando la escala B-O también fue normalizada en Barcelona (Gómez y Jiménez, 1972) con resultados 
semejantes a los de París (Borelli et Oléron, 1964) respecto a la capacidad del test de discriminar genéticamente (diferencias por edad y sexo) a los grupos estudiados: de 5 a 8 años de edad en París y de 4 a 6 años en Barcelona. De estos se tiene cuatro grupos comparables, los de 5 y 6 años de edad cuyos promedios en el total de la escala fueron los siguientes: en Barcelona, el grupo de 5 años, $\overline{\mathrm{x}}=21.43$ y de 6 años, $\overline{\mathrm{x}}=31.84$ y, en París, el grupo de 5 años, $\overline{\mathrm{x}}=21.55$ y de 6 años, $\bar{x}=32.47$.

Tratándose de una prueba de performance (Borelli et Oléron, 1964: 4) o de una escala de desarrollo (Gómez y Jiménez, 1972: 63 y 66), no se tienen reportes de la relación de esos resultados con el coeficiente de inteligencia (CI). Por ej., en el estudio con esa escala en personas con discapacidad intelectual no se encontró discriminación genética en las edades estudiadas (de 12 a 20 años de edad), pero sí una relación positiva del total en la escala B-O con el CI, $r=0.57(p=0.000)$, muy significativa, pero de intensidad moderada, por lo que se afirmó que ambos test miden funciones cognitivas ejecutivas, donde se entiende que comparten un buen porcentaje de la varianza (33\%) en esa dimensión de la inteligencia (Rivera, 2011).

Un antecedente relacionado en parte con nuestros objetivos es el que evaluó la capacidad de atención, funciones ejecutivas y memoria sobre el rendimiento académico (matemática y lenguaje) en un grupo de niños de segundo y sexto grado de primaria en la escuela pública de Guanajuato en Méjico (Castillo-Parra, Gómez Pérez y Ostrosky-Solís, 2009).

En esa investigación, se utilizó la batería neuropsicológica NEUROPSI Atención y memoria, con un redimensionamiento sustancial del Área II. Atención y concentración en una denominada "Área de Atención y Funciones Ejecutivas", según se pudo deducir de la referencia al instrumento, entre varias, por dos de sus autoras: Esmeralda Matute y Mónica Rosselli (Matute, Rosselli, Chamorro y Orozco, 2010: 133).

El instrumento de evaluación neuropsicológica NEUROPSI Atención y memoria 6 a 85 años (Ostrosky-Solís et al., 2003. Cit en Matute et al., 2010) cuenta con datos normativos para la población mejicana. Castillo-Parra et al. (2009) aplicaron el NEUROPSI en Guanajuato, con algunas variantes en la composición de sus áreas, subáreas e ítems, redimensionamiento del que dio cuenta Rivera (2013).

A diferencia del original en 2003, allí aparece una subárea Funciones ejecutivas, cuyos ítems, a nuestro entender, no son todos de su dominio, tales como formación de categorías, fluidez semántica y fonológica; ítems que son más bien del dominio del lenguaje y pensamiento; señalamiento que hacemos para tener en cuenta en la comparación de sus resultados con las funciones ejecutivas contenidas en IE y PC.

Aun en otro marco teórico, el estudio acerca de las inteligencias múltiples y el rendimiento escolar en el último año de la educación secundaria en Lima (Aliaga, Ponce, Bulnes, Elizalde, Montgomery, Gutiérrez, Delgado, Perea y Torchiani, 2012) lo referimos por su relación implícita con la inteligencia general y explíci- 
ta, en particular, con la inteligencia espacial, manteniendo la distancia, primero, respecto a la naturaleza de nuestra instrumentación (pruebas o test de ejecución para medir la IE y la PC, constructos del pensamiento espacial) y, luego, respecto a la población en ese estudio, jóvenes que podrían estar en un promedio entre 16 y 18 años de edad.

Veamos los argumentos acerca del sentido de la relación entre IE y PC:

Según Piaget, mediante el aprendizaje sensomotor, se logra construir el esquema corporal gracias al desarrollo de la inteligencia sensomotriz y los esquemas espaciales; luego, los procesos cognitivos, representativos figurales y operatorios del pensamiento y, según Luria, el esquema corporal se relaciona con el medio ambiente estructurando las representaciones espaciales mediante las estructuras sensomotoras que se ponen en juego, por lo que se genera así el pensamiento práxico constructivo. De este modo, el comportamiento espacial más general es el sensoriomotor.

Según Gérard de Montpellier, la adquisición del aprendizaje sensoriomotor apunta esencialmente a "(...) las relaciones entre los datos perceptivos o sensoriales y los procesos reactivos de naturaleza motriz. (...) Un ejemplo clásico de adquisición de nuevas coordinaciones sensoriomotrices se observa en el aprendizaje del dibujo en espejo" (De Montpellier, 1973: 69).

Nosotros consideramos necesario distinguir el aprendizaje sensoriomotor como proceso cognitivo que se da durante los dos primeros años de vida del hombre, etapa denominada por Piaget (1972) período sensoriomotor de la inteligencia, que comprende seis estadios. Dentro de estos, es a partir del estadio tres (4-8 meses de edad), llamado por Piaget estadio de las reacciones circulares secundarias, que se generan los aprendizajes sensomotores.

La diferencia entre los aprendizajes que refirió Piaget frente a los de De Montpellier radica en que, en el caso segundo, intervienen procesos cognitivos de carácter representacional, y en particular de los mecanismos psicogenéticos de las representaciones espaciales consolidada en la etapa adulta normal. También se sabe que, en el nivel del período de la inteligencia sensoriomotriz, el niño construye al nivel de la acción práctica y directa un espacio en el cual la organización está acabada, dispone de un conocimiento práctico que le permite hacer rodeos y componer los desplazamientos posibles y su capacidad de dominar las relaciones entre sus movimientos y los objetos se limita a los desplazamientos mismos en los que intervienen el control postural y la percepción, que no son funciones cognitivas representativas por no haber alcanzado los niveles operatorios del pensamiento que permiten los aprendizajes en los experimentos de De Montpellier, en términos de que el sujeto adulto ya posee nociones euclideanas del espacio.

Piaget, en La geometría espontánea del niño, demostró que aun los niños de 4 a 5 años tampoco pueden representarse un espacio conocido y, en Lógica y Conocimiento, comprobó que la representación gráfica de los niños (de 3 a 4 años) es de orden topológico. El dibujo nos aproxima a la comparación de los mecanismos 
cognitivos de carácter representacional del espacio (Piaget cit. en Droz y Rhamy, 1984: 66-8).

Este tipo de representación es característico del período preconcreto. Los dibujos no presentan relaciones simétricas ni proyectivas en su composición cuando se trata de formas concretas (personas, una familia, animales, flores, etc.). Los niños logran representaciones proyectivas y euclideanas más isomorfas con la realidad física en el período concreto de las operaciones (Piaget et Inhelder, 1972). Anotamos que los primeros dibujos son muy semejantes a los descritos por Luria (1977) en pacientes con agnosia óptica.

Pero antes de seguir a Luria, es oportuno señalar que la neurociencia, más recientemente, también ha estudiado cómo adquirimos la noción de espacio según Bryan Kolb e Ian Q. Whishaw (2006), quienes se basaron en observaciones clínicas realizadas en pacientes adultos con lesiones en los lóbulos temporal, parietal y frontal, que repercuten en la organización de la conducta espacial.

Kolb y Whishaw refieren los estudios de revisión de Aguirre y D' Esposito, quienes correlacionaron los distintos déficits de la orientación espacial con diferentes regiones del cerebro y las conductas espaciales relativas en el área del hemisferio derecho.

En cuanto al papel del lóbulo frontal, esos autores refirieron los experimentos en monos de R. K. Nakamura, R. Schein y R. Desimone y el aporte de R. Haaxma y H. Kuypers, ambos en 1986, que revelan una relación entre los déficits en la detección de objetos (indicadores visuales) y los déficits de la conducta espacial.

Por lo tanto, podemos afirmar que los estudios referidos hasta aquí por Kolb y Whishaw constituyen los correlatos neuropsicológicos vinculados al aprendizaje sensomotor de De Montpellier.

En cuanto a los aportes de la neuropsicología en el ámbito de la infancia, los estudios en la década del 2000 se refieren a las aplicaciones de resonancia magnética (RM) y resonancia magnética funcional (RMf) en niños. El camino tomado para establecer con más precisión las relaciones entre el desarrollo del cerebro y las capacidades cognitivas en edades tempranas consistió en poner de manifiesto la relación entre la teoría de los estadios del desarrollo cognitivo de Jean Piaget con los de los brotes de crecimiento, estudiados por H. T. Epstein en 1978: "Los primeros cuatro brotes de crecimiento coinciden con los cuatro estadios [períodos] principales del desarrollo cognitivo descritos por Piaget" (Kolb y Whishaw, 2006: 620).

Anotamos que Epstein estableció 5 períodos de los brotes del crecimiento cerebral entre los 3 y 10 meses a los 16 años de edad, de los cuales 4 brotes coinciden con los 4 períodos (no estadios) del desarrollo cognitivo. Según Piaget, esos períodos son: sensoriomotor, preconcreto, concreto y formal.

Asimismo, los autores señalaron cómo superar las dificultades de las correlaciones descritas. "Una forma de hacerlo es observar los intentos de un niño de resolver 
problemas específicos que son diagnósticos de lesión de regiones circunscritas del cerebro en los adultos" (Kolb y Whishaw, 2006: 621), como el estudiado por W. H. Overman y J. Bachevalier en 2001 sobre la función de los lóbulos temporales y los ganglios basales (cerebro anterior) en tareas de aprendizaje discriminativo en niños pequeños y adultos.

Coincidiendo con el planteamiento de Kolb y Whishaw para estudiar el desarrollo de los procesos cognitivos comparando adultos con patologías cerebrales y niños en relación con una tarea específica, ya David Rapaport (1965) señaló que, en la reorganización visual del espacio y reorganización de la acción motriz, sus operaciones y desplazamientos pueden pasar desapercibidos "(...) hasta que no se plantea alguna dificultad de carácter motor o visual (...)” (Rapaport, 1965: 45).

Así, veremos en lo que sigue cómo Luria afrontó el estudio de la praxis espacial a partir de estudios clínicos. Desde la neuropsicología, realizó estudios sistemáticos sobre las funciones práxicas como la alteración de la orientación en el espacio y la apractognosia constructiva, y denominó apractognosia a la menor o mayor perturbación de las formas más complejas de las síntesis óptico-espaciales, como son la pérdida de la orientación en el espacio y los fenómenos de apraxia espacial (Luria, 1977).

Él se apoya en los hallazgos de Piaget en 1935 para confirmar cuándo se plasma la orientación espacial y su ocurrencia en los lóbulos parieto-temporo-occipitales que intervienen en las funciones práxicas:

(...) en la composición de la orientación espacial se incluye la actividad práctica del niño, cuya posibilidad se forma al término del primer año de vida, al mismo tiempo que se consolida el trabajo conjunto de los analizadores visual, cinestésico y vestibular (N. M. Schelovánov, 1925; N. Figurin y M. P. Desínova, 1949) (Luria, 1977: 183).

Luria añadió al trabajo de los tres analizadores enumerados el hecho de que la percepción espacial del hombre se hace en el sistema de las coordenadas geométricas fundamentales (arriba/abajo, delante/atrás e izquierda/derecha) y dijo: "La forma más sencilla de pensamiento constructivo o práctico, que es familiar en medicina clínica, es la solución de tareas constructivas" (Luria, 1974: 328). Pero, luego de analizar la ejecución de esas tareas en pacientes con alteración de la síntesis espacial a causa de lesiones en las zonas parieto-occipitales del hemisferio izquierdo o con lesiones frontales, concluyó: "Estos hechos muestran que los procesos del pensamiento constructivo o práctico son de estructura compleja y tienen lugar a través de una serie de zonas del cerebro que trabajan de forma concertada" (Luria, 1974: 329-31).

En 1977, Luria propuso un proceso de pensamiento espacial que sobrepasa los límites de las funciones visuales superiores y que consiste en el análisis de las síntesis espaciales que son la base de la actividad constructiva y de las operaciones del pensamiento espacial. 
Como se ha sostenido, el proceso de pensamiento espacial en Luria y la evolución de las nociones de espacio en Piaget e Inhelder se complementan en la explicación de los mecanismos cognitivos requeridos para la solución de las tareas en los instrumentos de medición de las variables en el presente estudio. De esa forma, proponemos las siguientes definiciones conceptuales de las variables dependientes (constructos) inteligencia ejecutiva y praxia constructiva.

Inteligencia ejecutiva es una dimensión de la inteligencia general que consiste en la capacidad de resolver problemas prácticos de carácter espacio-temporal en los que intervienen las funciones visomotrices representacionales.

Praxia constructiva es, en términos neuropsicológicos, la capacidad de orientarse en el espacio por medio de la combinación de signos locales que provienen del aparato vestibular, kinestésico y visual, los cuales regulan el movimiento conjuntamente con la corteza sensomotora. Estas estructuras permiten los aprendizajes sensomotores complejos que constituyen la base para el análisis y las síntesis espaciales que dirigen la actividad constructiva y de las operaciones del pensamiento espacial.

Los resultados obtenidos aquí dicen que la IE se relaciona con la PC en muestras equiparadas (matching) por grado escolar (GE), sexo y nivel de rendimiento académico (NRA), variables independientes, que no produjeron diferencias en cada una de las funciones cognitivas referidas, diferencia que sí produjo la edad operatoria (concreto vs. preconcreto) en cada una de las muestras del estudio.

\section{MÉTODO}

\section{Tipo y diseño de investigación}

De enfoque cuantitativo, el estudio es de tipo no experimental. Respecto a la especificación de la tipología, esta es cuasi-transversal porque se realizó en dos momentos del año escolar (2013) por la necesidad de una segunda muestra semejante a la primera para establecer la asociación entre las escalas, la cual se obtuvo por matching en un contexto no experimental.

El diseño es correlacional porque se propuso averiguar si los resultados de la aplicación de las escalas B-O y BTd en una población escolar diferían o no según GE, sexo o NRA, además de averiguar el grado de asociación entre dichas escalas.

\section{Muestra}

El muestreo fue no probabilístico, y específicamente fue una "muestra intencional no probabilística de juicio" (Elorza, 2008: 185-6) debido a la necesidad, según los objetivos del estudio, de ciertas condiciones muestrales como edad, sexo y NRE según GE.

En total, participaron 54 escolares del primer al tercer grado de primaria en dos colegios de gestión privada, uno del distrito de Bellavista (Callao) y otro de Ca- 
rabayllo (Lima metropolitana), cuyas edades fueron de 6 años 1 mes a 9 años 4 meses $(\bar{x}=7.60, D E=0.89)$. La distribución por sexo fue de 26 hombres y 28 mujeres y, según el NRA, se obtuvo del nivel $\mathrm{AD}=13$, del $\mathrm{A}=40 \mathrm{y}$ del $\mathrm{B}=1$. En la tabla 1, se presenta el número de sujetos de la muestra 1 (Bellavista) y de la muestra 2 (Carabayllo), de acuerdo a la(s) escala(s) en la(s) que fueron evaluados.

Tabla 1. Total de sujetos evaluados con las escalas en las muestras

\begin{tabular}{cccc}
\hline Escala & Muestra 1 & Muestra 2 & Total \\
\hline B-O & 21 & - & 21 \\
B-O y BTd & 6 & - & 6 \\
BTd & - & 27 & 27 \\
Totales & 27 & 27 & 54 \\
\hline
\end{tabular}

En la muestra 1 (M1), se aplicó la escala B-O a 27 sujetos y la de BTd solo a 6 , donde se perdieron 21 sujetos, por lo cual se ubicó la muestra 2 (M2), en la que se aplicó la escala BTd en total también a 27 sujetos, con el objeto de obtener una muestra semejante a la M1 en GE, sexo y NRA.

En total, se realizaron 60 evaluaciones: 27 con el B-O (M1) y 33 con el BTd (6 en M1 y 27 en M2). Ellas se encuentran según GE en la tabla 2 y en la tabla 3 están los promedios de edad de los GE según muestra.

Tabla 2. Total de evaluaciones con las escalas en las muestras por grado escolar

\begin{tabular}{|c|c|c|c|c|}
\hline \multirow{2}{*}{ Grado escolar } & \multirow{2}{*}{$\begin{array}{c}\text { B-O } \\
\text { Muestra } 1\end{array}$} & \multicolumn{2}{|c|}{ BTd } & \multirow{2}{*}{ Tota } \\
\hline & & Muestra 1 & Muestra 2 & \\
\hline Primero & 9 & 2 & 9 & 20 \\
\hline Segundo & 9 & 0 & 9 & 18 \\
\hline Tercero & 9 & 4 & 9 & 22 \\
\hline Totales & 27 & 6 & 27 & 60 \\
\hline
\end{tabular}

Tabla 3. Promedios de edad en las muestras por grado escolar

\begin{tabular}{ccccccc}
\hline \multirow{2}{*}{ Grado escolar } & \multicolumn{3}{c}{ Muestra 1 } & \multicolumn{3}{c}{ Muestra 2 } \\
\cline { 2 - 7 } & $\mathrm{N}$ & $\overline{\mathrm{X}}$ & $\mathrm{DE}$ & $\mathrm{N}$ & $\overline{\mathrm{X}}$ & $\mathrm{DE}$ \\
\hline Primero & 9 & 6.49 & 0.30 & 9 & 6.69 & 0.31 \\
Segundo & 9 & 7.44 & 0.32 & 9 & 7.71 & 0.10 \\
Tercero & 9 & 8.69 & 0.31 & 9 & 8.58 & 0.32 \\
Totales & 27 & 7.54 & 0.96 & 27 & 7.66 & 0.86 \\
\hline
\end{tabular}




\section{INSTRUMENTOS}

Escala ejecutiva Borelli-Oléron - B-O (Borelli y Oléron, 2000)

Consta de siete pruebas (I a VII) o reactivos (ítems), seis de los cuales son conjuntos de objetos o material concreto donde la tarea del sujeto es armar (muñeco, encaje, rompecabezas o cubos) o copiar según el modelo o patrón que se le presente, y una, la prueba VI, consiste en copiar diseños geométricos según modelo impreso.

La puntuación máxima de la escala por sujeto puede ser 65 si no cometió ningún error en alguna de las pruebas, lo que le restaría puntos o fracción de punto. Las respuestas se califican con diferentes puntuaciones en cada prueba, de diferente grado de dificultad alternada, no sucesiva.

Las puntuaciones mínimas y máximas observadas por ítem fueron: 1 (3-14), 2 (3-5), 3 (4-10), 4 (0-7), 5 (7-11), 6 (4-10) y 7 (4-8).

\section{Test de praxia constructiva tridimensional - BTd (Benton, 2010)}

Consta de tres modelos (I a III) o reactivos (ítems) impresos que se presentan uno por vez al sujeto, que debe construirlos con un conjunto de 29 objetos de diferentes formas y dimensiones, del más fácil (modelo I) al más difícil (modelo III).

La puntuación máxima en esta escala puede ser 29 si el sujeto utilizó en cada modelo los objetos necesarios y en su justo lugar, de lo contrario se resta 1 punto por error. En el sistema estándar de puntuación los errores son de cuatro tipos: omisiones, adiciones, sustituciones y desplazamientos.

Las puntuaciones mínimas y máximas observadas por ítem fueron: 1 (3-6), 2 (07) y $3(0-15)$.

Procedimiento. Para lograr los objetivos y las pruebas de hipótesis se procedió como sigue:

1. Se definieron conceptualmente los constructos inteligencia ejecutiva (Piaget, 1972 y Piaget et Inhelder, 1972) y praxia constructiva (Luria, 1974, 1977), y se prepararon los protocolos para la aplicación de la escala ejecutiva Borelli-Oléron y el test de praxia constructiva tridimensional como definiciones operacionales (representantes) de los constructos a medir.

2. La recolección de los datos se llevó a cabo en dos colegios de gestión privada en la ciudad de Lima (Bellavista-Callao y Carabayllo-Lima metropolitana) durante el primer y segundo semestre, respectivamente, del año escolar 2013. Los permisos de acceso a los centros escolares (consentimiento informado) se obtuvieron a nivel institucional siendo administradas las pruebas por psicólogos, en sesiones de no menos de 45 minutos el B-O y 20 el Benton.

3. Los datos así recolectados se procesaron en el programa SPSS V. 17.0 para obtener los parámetros estadísticos y las normas de distribución. Como es de 
rigor, para el uso de estadísticos paramétricos (ej. $r$ de Pearson), se averiguó si las distribuciones de las puntuaciones obtenidas con las escalas no diferían significativamente de la poblacional con la prueba de Kolmogorov-Smirnov para cada muestra.

Esta prueba de ajuste no paramétrica "puede aplicarse a muestras pequeñas (...) únicamente procede para variables continuas (...) si los datos de la muestra se ajustan bien, la $\mathrm{D}_{\mathrm{MAX}}$ será significativamente pequeña (...) si el ajuste es pobre, será significativamente grande" (Elorza, 2008: 509-510).

\section{Validez y confiabilidad}

a) Para obtener la confiabilidad por consistencia interna de las escalas aplicadas, se utilizó el método de las 2 mitades Split-half según el coeficiente de predicción Spearman-Brown (S-B) como el más adecuado. Este método relaciona una mitad del test con la otra que al acortar el test disminuye la confiabilidad, de ahí la conveniencia de usar el coeficiente de predicción S-B, según el cual se estima la confiabilidad de longitud completa donde la correlación de las mitades se multiplica por el del test completo (Smith, 1975). En resumen, son autocorrelaciones que en mayor o menor grado nos informan sobre la confiabilidad. El coeficiente Spearman-Brown se recomienda en los test de ejecución, en lugar de formas paralelas (Garret, 1975).

El uso de la fórmula S-B se justifica también porque las escalas utilizadas se componen de pruebas o modelos (ítems) en número impar, 7 ítems el B-O y 3 el BTd, la mitad del BTd contiene solo un ítem necesariamente.

b) Otro criterio para considerar si, en la aplicación realizada, todos los ítems (pruebas en la escala B-O y modelos en el BTd) deberían permanecer en sus escalas fue el índice de homogeneidad (IH) del ítem. Es decir, qué tan semejantes son sus puntuaciones respecto al del test total según el coeficiente de correlación ítem-total corregido. Como criterio de aceptabilidad del ítem se fijó como mínimo el valor IH $=0.20$ (Ari, Jacobs y Razavich, 1999. Cit en Aliaga et al., 2012: 174).

c) Según el marco teórico, la validez de contenido de las escalas B-O y BTd comparten el rasgo común en la capacidad de discriminación genética por edad operatoria concreta y preconcreta.

Para conformar los grupos por edad operatoria se tomó aproximadamente el $25 \%$ de los extremos etarios en cada muestra en número de 6 sujetos, tanto para las submuestras de preconcretos como para la de concretos.

En el contraste de estos grupos por muestra, se utilizó la $t$ de Student para muestras independientes, previo test de Levene para determinar la igualdad o no de varianzas (homogeneidad), de donde se deriva el uso de la $t$. 
d) Como se vio en muestra (Tabla 1), en la M1, se obtuvo una submuestra ( $\mathrm{n}=$ 6) evaluada con ambas escalas, la cual se utilizó para averiguar acerca de la tendencia a la asociación de las escalas.

Primero, por el método gráfico, se averiguó si los perfiles de ejecución en la submuestra comportaban alguna semejanza o no con los obtenidos en las muestras totales, tanto en B-O como en BTd ( $\mathrm{N}=27$ en cada una), como indicio de la estabilidad de los constructos. Luego, para observar las asociaciones, dado el tamaño de la submuestra, se utilizó de manera exploratoria un coeficiente de correlación de rangos ordenados (rho de Spearman). Al menos, no hubo empates de puntuaciones en los totales, aunque sí en las pruebas del B-O y en los modelos del BTd.

Por lo expuesto, aún con la limitación muestral, para obtener el resultado final de las asociaciones entre escalas se usó el coeficiente de correlación de Pearson como prueba de validez concurrente. Pero, como de una aplicación a otra transcurrió un mes, ese resultado se puede tomar como prueba de una tendencia a la validez predictiva (Hernández et al., 2010: 202-3).

5. Las pruebas de hipótesis se realizaron primero para el contraste de los grupos, por GE, sexo y NRA en M1 y M2, y luego para la asociación de las escalas por intercorrelación.

a) El contraste de la diferencia de grupos se realizó con la prueba no paramétrica de análisis de varianza Kruskal-Wallis $(H)$, que sigue la distribución Chicuadrada $\left(x^{2}\right)$. Kruskal-Wallis es análoga al análisis de varianza cuando se usa con tres o más grupos (Elorza, 2008: 668-9) y, cuando son dos grupos, sería el equivalente al de una prueba $t$. También se usa en la comparación de grupos con n diferentes (Smith, 1975: 196-9).

De manera que esta prueba servirá en todos nuestros casos de comparación de grupos: dos para sexo, tres para GE y n diferentes en sexo y NRA por GE.

b) La asociación de la IE con la PC se realizó con la $r$ de Pearson entre las puntuaciones en las escalas por matching de M1 con M2.

Como señalamos en muestra y diseño, durante la aplicación del BTd, se perdieron por muerte experimental 21 de 27 sujetos en la M1 y como resultado se obtuvo una submuestra $(n=6)$ evaluada con ambas escalas. Según procedimiento en validez y confiabilidad (4.d), ese resultado sería auscultado gráfica y correlacionalmente como prueba de validez concurrente y estabilidad de las escalas.

En el método del matching 'emparejamiento', las asociaciones se realizan en dos muestras de sujetos diferentes utilizando un criterio para aparear a los sujetos según el mayor o menor rendimiento, por ejemplo, en una prueba de inteligencia o por NRA (Hernández et al., 2010: 134).

Según Reynaldo Alarcón, el objetivo esencial del diseño es conformar grupos equivalentes donde las variables de apareamiento pueden ser psicológicas, sociales y biológicas. Estadísticamente, se habrá conseguido la equivalencia entre los 
dos grupos cuando los promedios de esas variables no difieren significativamente (Alarcón, 2013: 111-9).

Entonces, además de las pruebas mencionadas en 4.d, será la hipótesis nula acerca de la diferencia de grupos que nos conducirá al matching por equivalencia de las muestras, siendo la variable biológica la principal variable de apareamiento en este caso.

\section{RESULTADOS}

\section{1) Estadística descriptiva}

a) En la tabla 4, se encuentran los estadísticos descriptivos de la aplicación de la escala B-O y la escala BTd por tipo de ítem y total en cada una de las escalas.

En esta, se observa que, en B-O, la mayoría de los ítems fueron elevados y solo la media del ítem 4 resultó más alejada de la puntuación total posible. En cambio, en BTd, solo el ítem 1 tuvo promedio elevado y los demás promedios fueron menores, lo que contribuyó a la tendencia a un nivel mayor de dificultad en la escala.

Tabla 4. Estadísticas descriptivas de las escalas B-O y BTd

\begin{tabular}{|c|c|c|c|c|c|c|c|c|}
\hline \multicolumn{9}{|c|}{$\mathrm{B}-\mathrm{O}$} \\
\hline & \multicolumn{7}{|c|}{ Tipos de ítems } & \multirow[b]{2}{*}{ Total } \\
\hline & 1 & 2 & 3 & 4 & 5 & 6 & 7 & \\
\hline Media & 11.11 & 4.70 & 8.59 & 3.74 & 9.19 & 8.39 & 6.87 & 52.59 \\
\hline Desviación estándar & 2.70 & 0.67 & 1.78 & 2.43 & 1.24 & 1.68 & 1.12 & 7.99 \\
\hline Error estándar medio & 0.52 & 0.13 & 0.34 & 0.47 & 0.24 & 0.32 & 0.22 & 1.54 \\
\hline Mínimo & 0.00 & 0.00 & 0.00 & 0.00 & 0.00 & 0.00 & 0.00 & 33.00 \\
\hline Máximo & 14.00 & 5.00 & 10.00 & 7.00 & 11.00 & 10.00 & 8.00 & 61.50 \\
\hline \multicolumn{9}{|c|}{ BTd } \\
\hline & & & 1 & 2 & 3 & Total & & \\
\hline Media & & & 5.48 & 4.15 & 7.82 & 17.44 & & \\
\hline Desviación estándar & & & 0.89 & 2.07 & 4.30 & 6.22 & & \\
\hline Error estándar medio & & & 0.17 & 0.40 & 0.83 & 1.20 & & \\
\hline Mínimo & & & 0.00 & 0.00 & 0.00 & 5.00 & & \\
\hline Máximo & & & 6.00 & 8.00 & 15.00 & 28.00 & & \\
\hline
\end{tabular}

Acerca de la normalidad en la distribución de las puntuaciones según la prueba Kolmogorov-Smirnov (K-S) con significación bilateral, se obtuvo $\mathrm{D}_{\mathrm{MAX}}=0.175$ en B-O $(p=0.381)$ y $\mathrm{D}_{\text {MAX }}=0.111(p=0.896)$ en BTd.

Según la distribución del estadístico K-S para $\mathrm{N}=27$ a un nivel de confianza de 0.05 , el valor crítico es igual a 0.254 . Las $\mathrm{D}_{\mathrm{MAX}}$ obtenidas son menores de 
0.254 , por lo que las distribuciones muestrales no difieren significativamente de la poblacional.

En la figura 1, vemos que las puntuaciones totales en B-O son elevadas, variaron de 33.00 a 61.50 con una media de 52.59 puntos $(\mathrm{DE}=7.99)$, donde 17 participantes $(63 \%)$ obtuvieron de 53.50 a 61.50 puntos, por encima de la media. También vemos que la distribución de las puntuaciones totales en BTd varía de 5.00 a 28.00 puntos con media de $17.44(\mathrm{DE}=6.22)$, donde 14 participantes (51.90\%) obtuvieron de 18 a 28 puntos, por encima de la media.

Figura 1. Histogramas de las puntuaciones totales de las escalas B-O y BTd

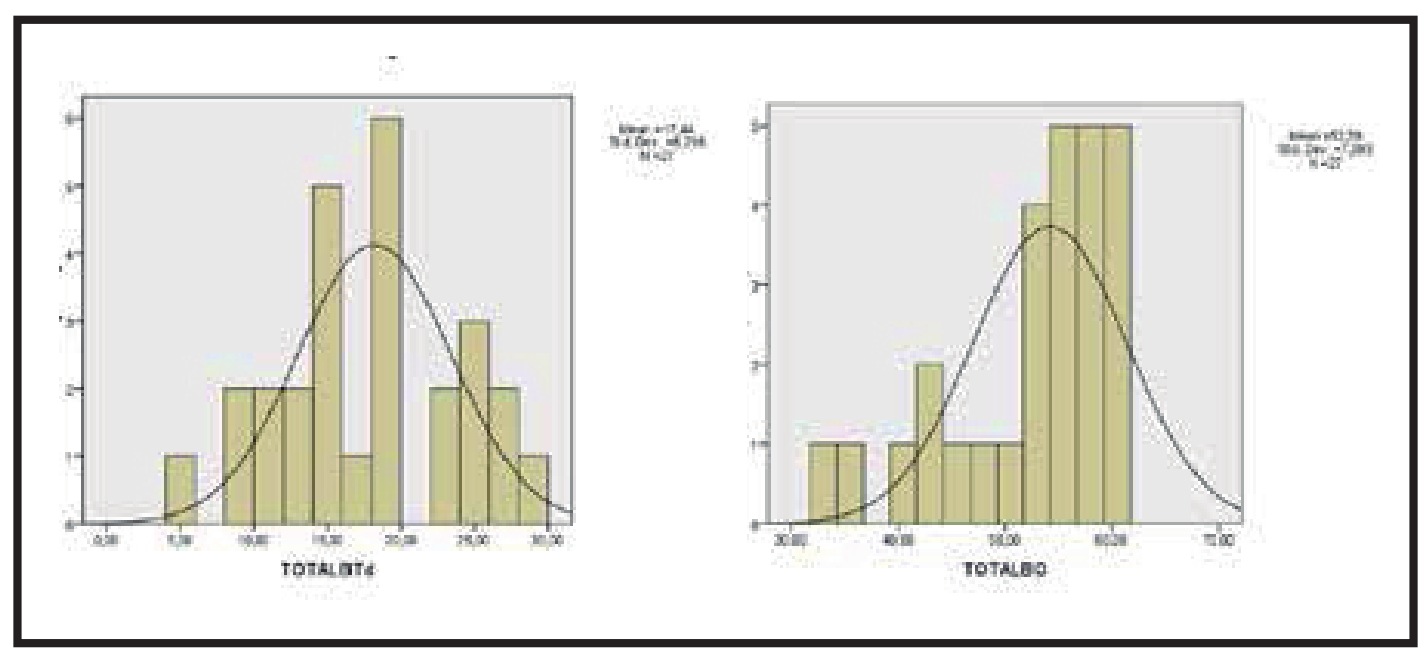

b) Para la comparación de las edades operatorias en cada muestra, se tomaron cuatro submuestras (de $n=6$ cada una), dos de preconcretos en primer grado escolar y dos de concretos en tercer grado. Es decir, a los menores de edad en las muestras les correspondió la edad operatoria preconcreta y, a los mayores, la concreta. En la tabla 5 se encuentran los promedios de edad en cada submuestra.

Tabla 5. Promedios de la edad cronólogica en las edades operatorias de las muestras

\begin{tabular}{lcc}
\hline & \multicolumn{2}{c}{ Promedio de edad cronológica } \\
\hline Edad operatoria & Muestra 1 & Muestra 2 \\
\hline Preconcreta & 6.33 & 6.50 \\
Concreta & 8.83 & 8.75 \\
\hline
\end{tabular}

En las edades operatorias de las muestras, las puntuaciones de los preconcretos en las escalas se distribuyeron de igual forma, 3 puntuaciones $(50 \%)$ por encima del promedio, $\overline{\mathrm{x}}=46.83$ en B-O y $\overline{\mathrm{X}}=14.83$ en BTd. En los concretos, fueron 4 puntuaciones $(67 \%)$ por encima de la $\bar{X}=56.92$ en $\mathrm{B}-\mathrm{O}$ y 5 puntuaciones (83\%) por encima de la $\bar{X}=22.83$ en BTd. Los demás parámetros descriptivos se encuentran en la siguiente tabla. 
Tabla 6. Estadísticas descriptivas de la escalas B-O y BTd según edad operatoria

\begin{tabular}{lcc}
\hline \multicolumn{2}{c}{ B-O } & \\
\hline & Edades operatorias \\
\hline Media & 46.83 & Concreta \\
Desviación estándar & 8.93 & 56.92 \\
Error estándar medio & 3.65 & 3.88 \\
Mínimo & 34.50 & 1.58 \\
Máximo & 58.00 & 50.00 \\
\hline & BTd & 61.50 \\
\hline & Preconcreta & Concreta \\
\hline Media & 14.83 & 22.83 \\
Desviación estándar & 3.19 & 6.49 \\
Error estándar medio & 1.30 & 2.65 \\
Mínimo & 11.00 & 10.00 \\
Máximo & 19.00 & 28.00 \\
\hline
\end{tabular}

c) La estadística descriptiva de la submuestra $(n=6)$ de M1, evaluada con ambas escalas, se presenta en la tabla 7 y resultó similar a las obtenidas con las muestras totales tanto en B-O como en BTd (tabla 4).

Tabla 7. Estadísticas descriptivas de las escalas B-O y BTd en submuestra $(n=6)$

\begin{tabular}{|c|c|c|c|c|c|c|c|c|}
\hline \multicolumn{9}{|c|}{$\mathrm{B}-\mathrm{O}$} \\
\hline & \multicolumn{7}{|c|}{ Tipos de ítems } & \multirow[b]{2}{*}{ Total } \\
\hline & 1 & 2 & 3 & 4 & 5 & 6 & 7 & \\
\hline Media & 11.08 & 4.83 & 8.83 & 4.50 & 9.08 & 8.67 & 7.17 & 54.17 \\
\hline Desviación estándar & 2.73 & 0.41 & 1.33 & 1.87 & 1.36 & 2.34 & 0.52 & 6.42 \\
\hline Error estándar medio & 1.11 & 0.17 & 0.54 & 0.76 & 0.55 & 0.96 & 0.21 & 2.62 \\
\hline Mínimo & 0.00 & 0.00 & 0.00 & 0.00 & 0.00 & 0.00 & 0.00 & 42.50 \\
\hline Máximo & 14.00 & 5.00 & 10.00 & 7.00 & 11.00 & 10.00 & 8.00 & 61.50 \\
\hline \multicolumn{9}{|c|}{ BTd } \\
\hline & & & 1 & 2 & 3 & Total & & \\
\hline Media & & & 5.67 & 5.17 & 9.83 & 20.67 & & \\
\hline Desviación estándar & & & 0.52 & 2.56 & 6.97 & 7.55 & & \\
\hline Error estándar medio & & & 0.21 & 1.05 & 2.85 & 3.08 & & \\
\hline Mínimo & & & 0.00 & 0.00 & 0.00 & 10.00 & & \\
\hline Máximo & & & 6.00 & 8.00 & 15.00 & 28.00 & & \\
\hline
\end{tabular}

Para ilustrar este hecho, graficamos los perfiles de ejecución observados en las puntuaciones de la escala BTd en M1 $(\mathrm{n}=6)$ con los obtenidos en $\mathrm{M} 2(\mathrm{~N}=27)$ (Figura 2). También se realizó lo mismo entre las puntuaciones de la escala B-O en M1 $(\mathrm{N}=27)$ y en su submuestra $(\mathrm{n}=6)$ (Figura 3$)$. 
Figura 2. Perfiles de ejecución observados (\%) en los modelos de la escala BTd en la muestra 2 $(\mathrm{N}=27)$ y en la submuestra $(\mathrm{n}=6)$ en relación con la puntuación máxima posible

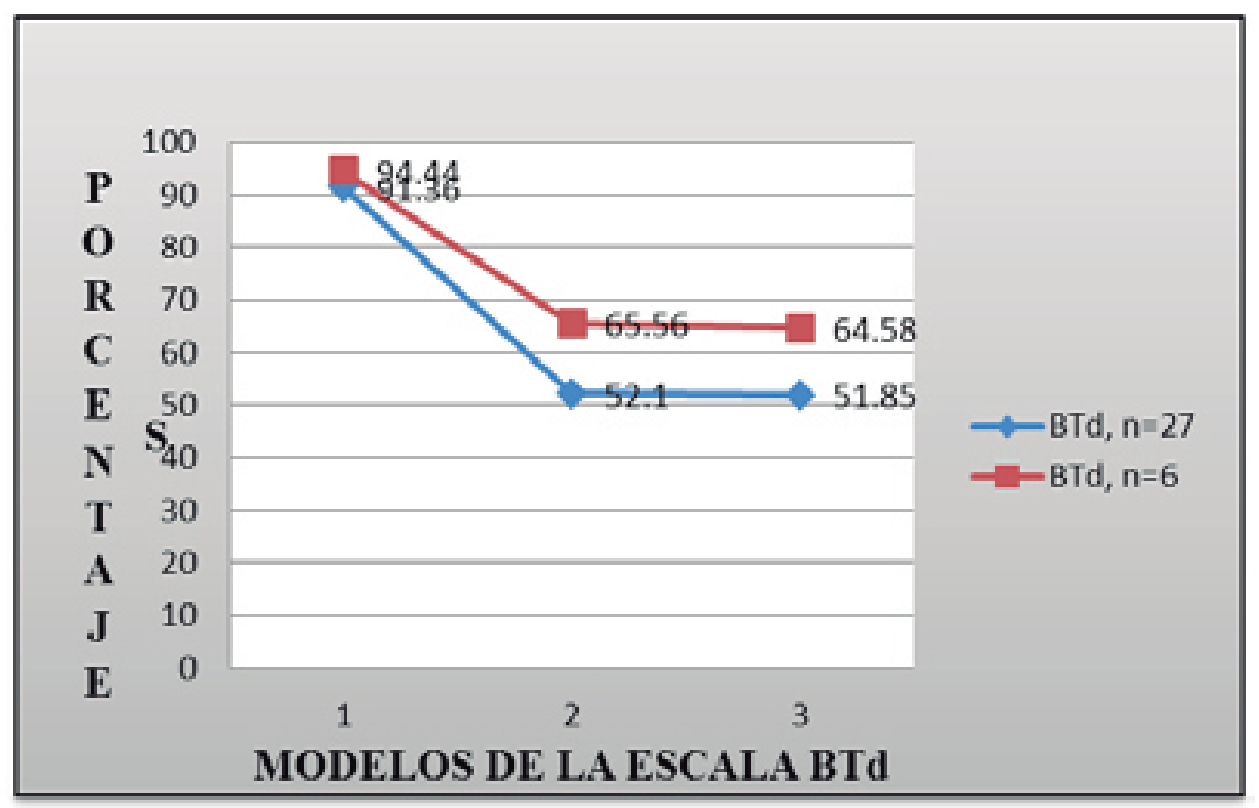

Figura 3. Perfiles de ejecución observados (\%) en las pruebas de la Escala B-O en la muestra 1 $(\mathrm{N}=27)$ y en su submuestra $(\mathrm{n}=6)$ en relación con la puntuación máxima posible

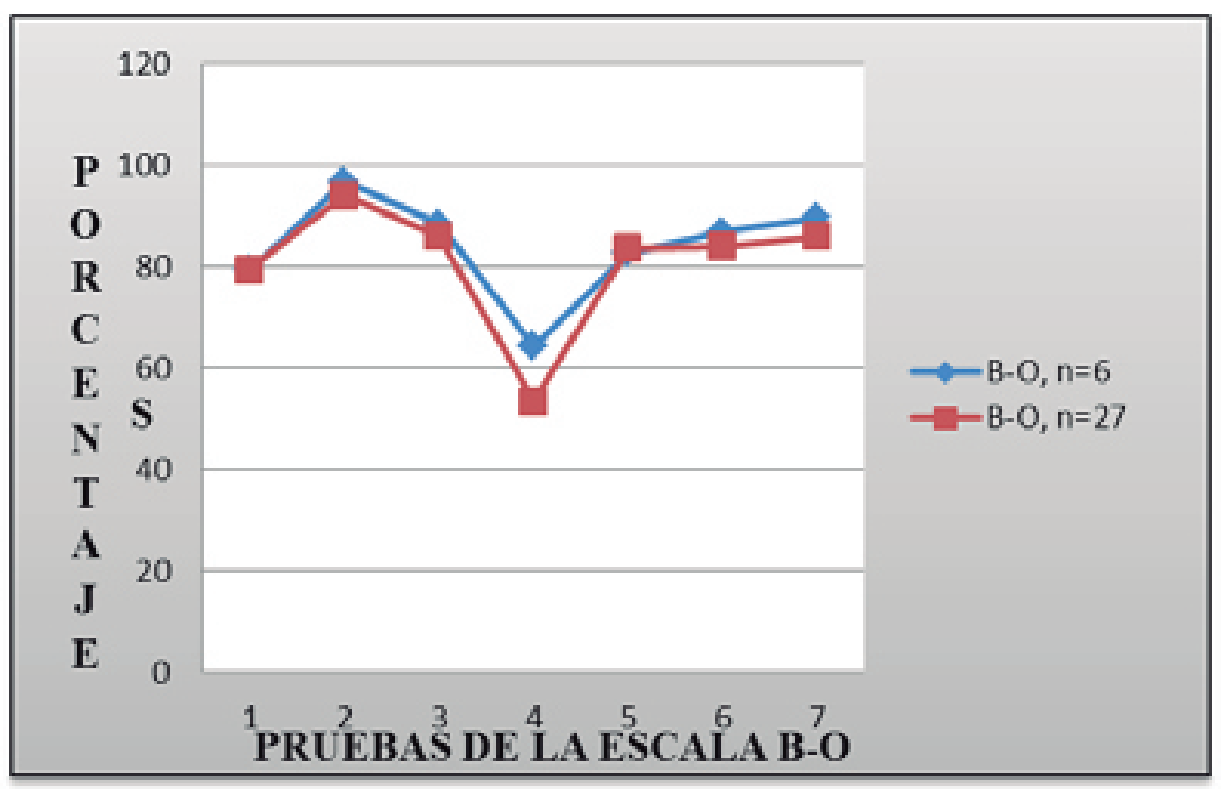

d) En las siguientes tablas se describen el comportamiento de las escalas B-O y BTd en la M1 y M2, respectivamente: según GE (tabla 8) y según sexo y NRA por GE (tablas 9 y 10), así como el total en cada escala con la excepción del NRA por GE en la M2 donde solo se obtuvo un GE (segundo) con más de un nivel de rendimiento escolar ( $\mathrm{AD}$ y A) para efectos de la comparación de grupos. En esta muestra todos los del primer y tercer grado escolar fueron del nivel A (96 \% en la muestra total). 
Tabla 8. Estadísticas descriptivas de las escalas B-O en M1 y BTd en M2 según GE

\begin{tabular}{|c|c|c|c|c|}
\hline \multicolumn{5}{|c|}{$\mathrm{B}-\mathrm{O}$} \\
\hline Grado escolar & Primero & Segundo & Tercero & Total \\
\hline $\mathrm{N}$ & 9 & 9 & 9 & 27 \\
\hline Media & 49.61 & 54.44 & 53.72 & 52.59 \\
\hline Desviación estándar & 8.41 & 7.10 & 8.43 & 7.99 \\
\hline Error estándar medio & 2.81 & 2.37 & 2.81 & 1.54 \\
\hline Mínimo & 34.50 & 41.00 & 33.00 & 33.00 \\
\hline Máximo & 59.50 & 61.50 & 61.50 & 61.50 \\
\hline \multicolumn{5}{|c|}{ BTd } \\
\hline Grado escolar & Primero & Segundo & Tercero & Total \\
\hline $\mathrm{N}$ & 9 & 9 & 9 & 27 \\
\hline Media & 14.56 & 17.78 & 20.00 & 17.44 \\
\hline Desviación estándar & 3.58 & 4.79 & 8.54 & 6.22 \\
\hline Error estándar medio & 1.19 & 1.60 & 2.85 & 1.20 \\
\hline Mínimo & 9.00 & 9.00 & 5.00 & 5.00 \\
\hline Máximo & 19.00 & 25.00 & 28.00 & 28.00 \\
\hline
\end{tabular}

Tabla 9. Estadísticas descriptivas de la escala B-O en M1 y de la escala BTd en M2 según sexo por GE

\begin{tabular}{|c|c|c|c|c|c|c|c|}
\hline \multicolumn{8}{|c|}{$\mathrm{B}-\mathrm{O}$} \\
\hline Grado escolar & \multicolumn{2}{|c|}{ Primero } & \multicolumn{2}{|c|}{ Segundo } & \multicolumn{2}{|c|}{ Tercero } & \multirow{2}{*}{ Total } \\
\hline Sexo & $\mathrm{H}$ & M & $\mathrm{H}$ & M & $\mathrm{H}$ & M & \\
\hline $\mathrm{N}$ & 4 & 5 & 4 & 5 & 5 & 4 & 27 \\
\hline Media & 49.00 & 50.10 & 58.38 & 51.30 & 55.80 & 51.13 & 52.59 \\
\hline Desviación estándar & 10.69 & 7.44 & 3.22 & 8.08 & 4.37 & 12.17 & 7.99 \\
\hline Error estándar medio & 5.34 & 3.33 & 1.61 & 3.61 & 1.95 & 6.08 & 1.54 \\
\hline Mínimo & 34.50 & 42.50 & 54.50 & 41.00 & 50.00 & 33.00 & 33.00 \\
\hline Máximo & 58.00 & 59.50 & 61.50 & 61.50 & 61.50 & 59.00 & 61.50 \\
\hline \multicolumn{8}{|c|}{ BTd } \\
\hline Grado escolar & \multicolumn{2}{|c|}{ Primero } & \multicolumn{2}{|c|}{ Segundo } & \multicolumn{2}{|c|}{ Tercero } & Totol \\
\hline Sexo & $\mathrm{H}$ & M & $\mathrm{H}$ & M & $\mathrm{H}$ & M & Total \\
\hline $\mathrm{N}$ & 5 & 4 & 4 & 5 & 4 & 5 & 27 \\
\hline Media & 13.00 & 16.50 & 17.75 & 17.80 & 19.75 & 20.20 & 17.44 \\
\hline Desviación estándar & 3.54 & 2.89 & 4.11 & 5.76 & 9.91 & 8.50 & 6.22 \\
\hline Error estándar medio & 1.58 & 1.44 & 2.06 & 2.58 & 4.96 & 3.80 & 1.20 \\
\hline Mínimo & 9.00 & 14.00 & 14.00 & 9.00 & 5.00 & 10.00 & 5.00 \\
\hline Máximo & 18.00 & 19.00 & 23.00 & 25.00 & 26.00 & 28.00 & 28.00 \\
\hline
\end{tabular}


Tabla 10. Estadísticas descriptivas de la escala BTd en M2 y de la escala B-O en M1 según NRA por GE

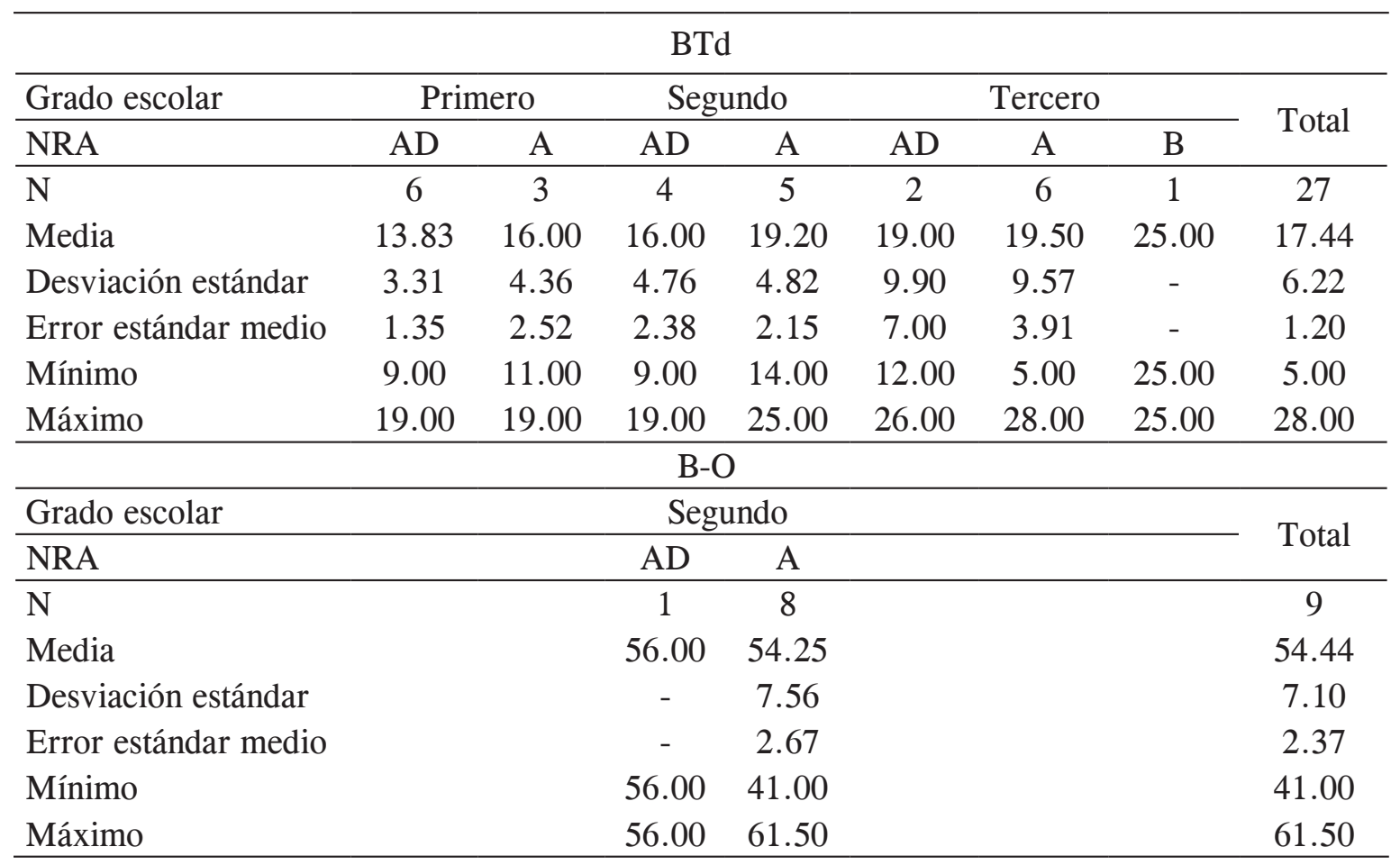

\section{Validez y confiabilidad}

a) El resultado del coeficiente de predicción Spearman-Brown, utilizado para estimar la confiabilidad por consistencia interna, fue 0.87 en la escala B-O y 0.81 en la escala BTd.

Según Milton Smith, “(...) si una prueba tiene por objeto diferenciar entre las medias de dos o más grupos, una confiabilidad de 0.80 es adecuada” (Smith, 1975: 155-6).

b) El índice de homogeneidad (IH) de los ítems en las escalas (Tabla 11) fue de 0.26 a 0.69 en la escala B-O y de 0.31 a 0.68 en la escala BTd. Por lo tanto, de acuerdo al mínimo aceptable adoptado $(\mathrm{IH}=0.20)$ fueron consideradas las 7 pruebas del B-O y los 3 modelos del BTd.

Tabla 11. Índice de homogeneidad en las escalas B-O y BTd

\begin{tabular}{|c|c|c|c|c|c|c|}
\hline \multicolumn{7}{|c|}{$\mathrm{B}-\mathrm{O}$} \\
\hline \multicolumn{7}{|c|}{ Tipos de ítems } \\
\hline 1 & 2 & 3 & 4 & 5 & 6 & 7 \\
\hline 0.52 & 0.55 & 0.50 & 0.58 & 0.26 & 0.59 & 0.69 \\
\hline \multicolumn{7}{|c|}{ BTd } \\
\hline & & 1 & 2 & 3 & & \\
\hline & & 0.31 & 0.65 & 0.68 & & \\
\hline
\end{tabular}


c) Los resultados acerca de la capacidad de discriminación de las escalas según edad operatoria en cada muestra fueron significativos, tal como aparecen en la Tabla 12.

Tabla 12. Diferencias de medias en las escalas B-O y BTd por edad operatoria según la t de Student

\begin{tabular}{|c|c|c|}
\hline \multicolumn{3}{|c|}{$\mathrm{B}-\mathrm{O}$} \\
\hline & Concreto & Preconcreto \\
\hline $\mathrm{n}$ & 6 & 6 \\
\hline Diferencia de medias & \multicolumn{2}{|c|}{10.08} \\
\hline Error estándar de la diferencia & \multicolumn{2}{|c|}{3.98} \\
\hline $\mathrm{t}$ & \multicolumn{2}{|c|}{2.54} \\
\hline Grados de libertad & \multicolumn{2}{|c|}{10} \\
\hline Significación bilateral & \multicolumn{2}{|c|}{0.03} \\
\hline \multicolumn{3}{|l|}{ BTd } \\
\hline & Concreto & Preconcreto \\
\hline $\mathrm{n}$ & 6 & 6 \\
\hline Diferencia de medias & \multicolumn{2}{|c|}{8.00} \\
\hline Error estándar de la diferencia & \multicolumn{2}{|c|}{2.95} \\
\hline $\mathrm{t}$ & \multicolumn{2}{|c|}{2.71} \\
\hline Grados de libertad & \multicolumn{2}{|c|}{10} \\
\hline Significación bilateral & \multicolumn{2}{|c|}{0.02} \\
\hline
\end{tabular}

Estos resultados aportan a la validez de contenido de las escalas que atiende a la hipótesis teórica acerca de la relación en los presupuestos que fundamentan sus constructos.

d) Dadas las características de las puntuaciones en las escalas B-O y BTd, donde se presentaron varios empates, lo cual disminuye la potencia en los coeficientes de correlación por rangos ordenados, se usó de manera exploratoria la Rho de Spearman $\left(r_{s}\right)$ en la submuestra $(\mathrm{n}=6)$ de $\mathrm{M} 1$, evaluada con ambas escalas, y se encontró $r_{s}=0.60$, no significativa $(p=0.21)$.

La no significación estadística se atribuye a la $n$ pequeña, pero el valor absoluto se ubica en el intervalo de correlación moderada (Elorza, 2008: 468). Esto nos informa que las escalas comparten un porcentaje importante de la varianza (36\%).

Luego, con el coeficiente de correlación de Pearson $r=0.84(p=0.039)$, el valor absoluto subió al intervalo de correlación significativa, con lo cual se obtuvo una prueba de validez concurrente de las escalas B-O y BTd en la submuestra.

Es oportuno referir aquí las figuras 2 y 3 ya vistas por la hipótesis acerca de la estabilidad de las escalas en una submuestra y su muestra mayor en B-O (figura 3 ), y en esa submuestra y una muestra mayor en BTd (figura 2) que no son de los mismos sujetos ni del mismo colegio, pero que son semejantes por GE, sexo y NRA. Estos resultados suman y aportan a la validez de constructo de las escalas aplicadas. 
Hasta aquí, se han obtenido pruebas de validez y confiabilidad de la aplicación de las escalas utilizadas en el estudio como representantes (definiciones operacionales) de la inteligencia ejecutiva (Escala B-O) y de la praxia constructiva (Escala BTd). A la validez de constructo, principalmente demostrada por la validez concurrente y de contenido, se le suma la confiabilidad por consistencia interna tanto en sus indicadores totales como en cada uno de sus ítems.

3. Los resultados en las pruebas de hipótesis fueron los siguientes:

a) Diferencia de grupos. En las siguientes tablas, se encuentran los resultados obtenidos con la prueba Kruskal-Wallis en M1 (Tabla 13) y en M2 (Tabla 14). Se encontró que tanto la escala B-O como la escala BTd no se diferencian por el GE ni por el sexo y el NRA por GE. Así, las $x^{2}$ fueron muy pequeñas ( 0.05$)$ o nulas $(p=1.00)$, por lo que no se pudo rechazar la hipótesis nula en ningún caso.

Tabla 13. Diferencias de la escala B-O en M1 según GE y sexo y NRA por GE, según la prueba Kruskal-Wallis

\begin{tabular}{|c|c|c|c|c|c|c|}
\hline Grado escolar & \multicolumn{2}{|c|}{ Primero } & \multicolumn{2}{|c|}{ Segundo } & \multicolumn{2}{|c|}{ Tercero } \\
\hline $\mathrm{N}$ & \multicolumn{2}{|c|}{9} & \multicolumn{2}{|c|}{9} & \multicolumn{2}{|c|}{9} \\
\hline Rango medio & \multicolumn{2}{|c|}{11.00} & \multicolumn{2}{|c|}{15.72} & \multicolumn{2}{|c|}{15.28} \\
\hline Chi-cuadrada & \multicolumn{6}{|c|}{1.950} \\
\hline Grados de libertad & \multicolumn{6}{|c|}{2} \\
\hline Significación bilateral & \multicolumn{6}{|c|}{0.377} \\
\hline Grado escolar & \multicolumn{2}{|c|}{ Primero } & \multicolumn{2}{|c|}{ Segundo } & \multicolumn{2}{|c|}{ Tercero } \\
\hline Sexo & $\mathrm{H}$ & $\mathrm{M}$ & $\mathrm{H}$ & $\mathrm{M}$ & $\mathrm{H}$ & $\mathrm{M}$ \\
\hline $\mathrm{N}$ & 4 & 5 & 4 & 5 & 5 & 4 \\
\hline Rango medio & 5.00 & 5.00 & 6.38 & 3.90 & 5.20 & 4.75 \\
\hline Chi-cuadrada & \multicolumn{2}{|c|}{0.000} & \multicolumn{2}{|c|}{1.830} & \multicolumn{2}{|c|}{0.060} \\
\hline Grados de libertad & \multicolumn{2}{|c|}{1} & \multicolumn{2}{|c|}{1} & \multicolumn{2}{|c|}{1} \\
\hline Significación bilateral & \multicolumn{2}{|c|}{1.000} & \multicolumn{2}{|c|}{0.176} & \multicolumn{2}{|c|}{0.806} \\
\hline Grado escolar & \multicolumn{6}{|c|}{ Segundo } \\
\hline NRA & \multicolumn{4}{|c|}{$\mathrm{AD}$} & & \\
\hline $\mathrm{N}$ & \multicolumn{4}{|c|}{1} & & \\
\hline Rango medio & & & 5.00 & 5.00 & & \\
\hline Chi-cuadrada & \multicolumn{6}{|c|}{0.000} \\
\hline Grados de libertad & \multicolumn{6}{|c|}{1} \\
\hline Significación bilateral & \multicolumn{6}{|c|}{1.000} \\
\hline
\end{tabular}


Tabla 14. Diferencias de la escala BTd en M2 según GE y sexo y NRA por GE, según la prueba Kruskal-Wallis

\begin{tabular}{|c|c|c|c|c|c|c|c|}
\hline Grado escolar & \multicolumn{2}{|c|}{ Primero } & \multicolumn{2}{|c|}{ Segundo } & \multicolumn{2}{|c|}{ Tercero } & \\
\hline $\mathrm{N}$ & \multicolumn{2}{|c|}{9} & \multicolumn{2}{|c|}{9} & \multicolumn{2}{|c|}{9} & \\
\hline Rango medio & \multicolumn{2}{|c|}{10.17} & \multicolumn{2}{|c|}{14.50} & \multicolumn{2}{|c|}{17.33} & \\
\hline Chi-cuadrada & \multicolumn{6}{|c|}{3.760} & \\
\hline Grados de libertad & \multicolumn{6}{|c|}{2} & \\
\hline Significación bilateral & \multicolumn{6}{|c|}{0.153} & \\
\hline Grado escolar & \multicolumn{2}{|c|}{ Primero } & \multicolumn{2}{|c|}{ Segundo } & \multicolumn{2}{|c|}{ Tercero } & \\
\hline Sexo & $\mathrm{H}$ & $\mathrm{M}$ & $\mathrm{H}$ & $\mathrm{M}$ & $\mathrm{H}$ & $\mathrm{M}$ & \\
\hline $\mathrm{N}$ & 5 & 4 & 4 & 5 & 4 & 5 & \\
\hline Rango medio & 3.80 & 6.50 & 4.75 & 5.20 & 4.50 & 5.40 & \\
\hline Chi-cuadrada & \multicolumn{2}{|c|}{2.197} & \multicolumn{2}{|c|}{0.062} & \multicolumn{2}{|c|}{0.244} & \\
\hline Grados de libertad & \multicolumn{2}{|c|}{1} & \multicolumn{2}{|c|}{1} & \multicolumn{2}{|c|}{1} & \\
\hline Significación bilateral & \multicolumn{2}{|c|}{0.138} & \multicolumn{2}{|c|}{0.803} & \multicolumn{2}{|c|}{0.621} & \\
\hline Grado escolar & \multicolumn{2}{|c|}{ Primero } & \multicolumn{2}{|c|}{ Segundo } & \multicolumn{3}{|c|}{ Tercero } \\
\hline NRA & $\mathrm{AD}$ & A & $\mathrm{AD}$ & A & $\mathrm{AD}$ & A & $\mathrm{B}$ \\
\hline $\mathrm{N}$ & 6 & 3 & 4 & 5 & 2 & 6 & 1 \\
\hline Rango medio & 4.58 & 5.83 & 4.25 & 5.60 & 5.25 & 4.83 & 5.5 \\
\hline Chi-cuadrada & \multicolumn{2}{|c|}{0.424} & \multicolumn{2}{|c|}{0.559} & \multicolumn{3}{|c|}{0.073} \\
\hline Grados de libertad & \multicolumn{2}{|c|}{1} & \multicolumn{2}{|c|}{1} & \multicolumn{3}{|c|}{2} \\
\hline Significación bilateral & \multicolumn{2}{|c|}{0.515} & \multicolumn{2}{|c|}{0.455} & & & \\
\hline
\end{tabular}

b) Intercorrelación de escalas. El matching realizado bien podría denominarse intencionado porque, además de no enmarcarse en una investigación experimental (causal o predictiva), utiliza por defecto las variables controladas (GE, sexo, NRA) post facto, es decir, después que actuaron, no antes. Tal es el caso de la hipótesis nula $\left(\mathrm{H}_{0}\right)$, acerca de las diferencias de grupos en el comportamiento de las escalas, vista en la sección anterior (3.a).

Al no poder rechazar la $\mathrm{H}_{0}$ en ningún caso, la conclusión es que los grupos en cada muestra provienen de la misma población. Solo queda probar, no sin antes analizar los criterios según variable observada y la edad cronológica (variable biológica) del match, es decir, cómo se conformó cada par de sujetos (27 pares en total).

- Si bien no existe diferencia de grupos por GE en M1 (Tabla 13) y en M2 (Tabla 14) para el emparejamiento de las muestras, se conservó esta unidad de observación por encontrarse claramente la tendencia, aunque no significativa, que a mayor GE mayor rango medio en la escala BTd.

- Por el sexo, existe la tendencia no significativa en ambas muestras, pero, en M1, se ubica solo en el segundo GE (Tabla 13) y en M2 solo se ubica en el 
primer GE (Tabla 14). Por lo tanto, el sexo no se consideró un criterio de emparejamiento por ser indistinto para la mayoría de GE.

- El NRA sin diferencia significativa por GE equiparó la M2 con la M1. En M2, el único sujeto de nivel B no produjo distinción alguna en el tercer GE (Tabla 14). En M1, sucedió lo mismo con el único sujeto $\mathrm{AD}$ en el segundo GE (Tabla 13). El nivel A en M1 fue el $96 \%$.

- Dentro de cada GE los pares se conformaron entre los de igual edad (años/ meses) o con la edad más próxima. En casos de empate de edad, en una u otra muestra, se usó la tendencia a la correlación positiva entre las puntuaciones totales en las escalas (Resultado 1.c y 2.d) para elegir al match 'par'.

Entonces, del primer al tercer grado en M1 y M2, los pares del primer grado se formaron con los sujetos de ambas muestras en ese grado (grupo de pares del 1 al 9), luego, los pares del segundo grado (son los pares del 10 al 18) y, por último, los pares del tercer grado (pares del 19 al 27). Obviamente, el resultado del procedimiento descrito es el conjunto de 27 pares de puntuaciones que los sujetos emparejados obtuvieron en la escala correspondiente, objeto de la prueba de intercorrelación.

Como prueba de asociación de las escalas B-O y BTd, se calcularon los coeficientes de correlación de Pearson entre los ítems de cada una, así como entre los ítems y total por escala (Tabla 15). La mayoría de las correlaciones fueron positivas (31 de 32 coeficientes en total), entre significativas y muy significativas (15 de 32) y de magnitud débil y moderada.

De las correlaciones no significativas (17 en 32 coeficientes), 4 fueron nulas y las demás muy débiles. En esta categoría, se ubica la única correlación negativa, entre la prueba 5 de la escala B-O y la 1 de la escala BTd. Esa prueba, junto con la 2 y la 6 del B-O, no correlaciona con ningún modelo del BTd, pero la 6 correlaciona con el total.

Con excepción de la correlación entre la prueba 5 y el total BTd (correlación muy débil), las correlaciones entre las puntuaciones totales del B-O y del BTd variaron de 0.27 a 0.61 .

Cabe destacar que la mayoría de las pruebas del B-O (5 de 7) correlacionan significativamente con el total BTd, y la mayoría de las pruebas del BTd (2 de 3) correlacionan significativamente con el total B-O. 
Tabla 15. Correlaciones de Pearson entre las escalas B-O y BTd

\begin{tabular}{|c|c|c|c|c|c|c|}
\hline & & & \multicolumn{4}{|c|}{ Escala BTd } \\
\hline & & & 1 & 2 & 3 & Total \\
\hline \multirow{14}{*}{$\begin{array}{c}\text { Escala } \\
\text { B-O }\end{array}$} & \multirow{2}{*}{1} & $r$ & 0.009 & 0.438 & 0.410 & 0.430 \\
\hline & & $p$ & 0.965 & 0.022 & 0.034 & 0.025 \\
\hline & \multirow{2}{*}{2} & $r$ & 0.055 & 0.339 & 0.288 & 0.320 \\
\hline & & $p$ & 0.786 & 0.084 & 0.145 & 0.104 \\
\hline & \multirow{2}{*}{3} & $r$ & 0.418 & 0.507 & 0.532 & 0.597 \\
\hline & & $p$ & 0.030 & 0.007 & 0.004 & 0.001 \\
\hline & \multirow{2}{*}{4} & $r$ & 0.308 & 0.528 & 0.368 & 0.474 \\
\hline & & $p$ & 0.118 & 0.005 & 0.059 & 0.012 \\
\hline & \multirow{2}{*}{5} & $r$ & -0.136 & 0.213 & 0.017 & 0.064 \\
\hline & & $p$ & 0.500 & 0.285 & 0.931 & 0.752 \\
\hline & \multirow{2}{*}{6} & $r$ & 0.331 & 0.297 & 0.377 & 0.407 \\
\hline & & $p$ & 0.092 & 0.132 & 0.052 & 0.035 \\
\hline & \multirow{2}{*}{7} & $r$ & 0.160 & 0.480 & 0.318 & 0.402 \\
\hline & & $p$ & 0.424 & 0.011 & 0.106 & 0.037 \\
\hline \multirow{2}{*}{\multicolumn{2}{|c|}{ Total }} & $r$ & 0.266 & 0.613 & 0.520 & 0.601 \\
\hline & & $p$ & 0.181 & 0.001 & 0.005 & 0.001 \\
\hline
\end{tabular}

\section{DISCUSIÓN}

Visto en antecedente, en muestra y en resultado descriptivo (1.a) de la aplicación de la escala B-O en M1, del primer al tercer grado escolar (aproximados a la edad cronológica de 6 a 8 años respectivamente), los grupos comparables con París y Barcelona son los siguientes: con París, los de 6 y 8 años y con Barcelona, el de 6 años. Esto, siempre y cuando se equipare los rangos de edad a 3 meses antes o después de la fecha de nacimiento por edad, tal como se hizo en París y, al parecer, también en Barcelona, salvando las distancias con los tamaños de esas muestras, pues en nuestro caso distan enormemente $(\mathrm{n}<30)$.

Las muestras equiparadas ( $a d$ hoc) son submuestras de los grados escolares: en 6 años es submuestra de los preconcretos, por lo tanto, del primer GE; y en 8 años, al comprender a niños de 7 también, incluye a niños del segundo y tercer GE (en las muestras del estudio los GE son multiedad) y no son submuestra de los concretos (estos son los mayores del tercer GE).

Así, el promedio de la puntuaciones totales de la escala B-O en la submuestra ad hoc de 6 años de edad en Lima es $\bar{x}=45.00$, ligeramente menor que en la edad operatoria preconcreta cuya $\bar{x}=46.83$ (Tabla 6), pero es mucho mayor que en París, $\bar{x}=32.47$, y que en Barcelona, $\bar{x}=31.84$. 
De manera que, luego de casi cinco décadas (1964-2013), aquí tendríamos una ganancia generacional en IE, un promedio de 3 puntos de ganancia por década. “(...) para las naciones que ya eran desarrolladas a principios del siglo XX, la tasa ha sido de 3 puntos de CI de ganancia por década" (Flynn, 1987. Cit en León, 2012: 227).

Sin embargo, esta ganancia sería menor en los niños de 8 años de edad en Lima (submuestra ad hoc), cuyo promedio en el total $\mathrm{B}-\mathrm{O}, \overline{\mathrm{X}}=50.83$, es solo 5 puntos más que en París, $\overline{\mathrm{x}}=45.87$.

La pregunta es si esta menor ganancia generacional en los de 8 años de edad respecto a los de 6 años solo sucede con la IE en la población escolar básica o también sucede con el CI, pues el argumento de Flynn en 2009 acerca de las causas en los incrementos intelectuales (revolución industrial y desarrollo de una actitud científica y analítica en la población) “(...) es rebatido por la demostración de impresionantes ganancias generacionales entre niños preescolares (...)" (Lynn, 2009. Cit en León, 2012: 227).

Respecto a la escala BTd, para su calificación utilizamos el sistema estándar de puntuación según su manual (Benton, 1968) y se observó mayor dificultad de ejecución en los modelos 2 y 3 , responsables de una no muy alta performance en el total del test en Lima. En ese entonces, el autor del test tuvo una suposición interesante según a los hallazgos de Domrath en 1965 (Iowa-EUA) en niños de inteligencia media entre 7 y 1 mes a 8 años de edad, donde el $77 \%$ de la muestra no tuvo ningún error, o sea que puntuaron 29 (el resto tuvo entre 2 y 6 errores).

Benton comparó el resultado de Domrath, quien utilizó el segundo sistema de puntuación (que solo considera omisiones y sustituciones y no adiciones o desplazamientos de uno o varios bloques como errores), con los hallados por él en adultos normales, con el sistema estándar, utilizando el segundo sistema de puntuación como en Domrath, y señaló: "Si la mayoría de sujetos de 7 a 8 años no tienen ningún error, se puede suponer razonablemente que el nivel de éxito adulto debe ser alcanzado a la edad de 9 años" (Benton, 1968, p. 13).

Bien, nosotros no hemos procesado la data con el segundo sistema de puntuación para poder extrapolar tanto los resultados obtenidos por Domrath como la suposición de Benton, es decir, que los niños deben obtener el éxito total en el test a los 9 años de edad.

Anotamos que en Lima el grupo comparable al de Iowa en Domrath sería el de segundo GE en la M2, cuyo promedio de edad fue 7.71 (Tabla 3) y el grupo comparable al hipotético caso de Benton sería el de la edad operatoria concreta en la misma M2, cuyo promedio de edad fue 8.75 (Tabla 5) cerca a 8 y 9 años de edad respectivamente.

Según el NEUROPSI, test neuropsicológico que evalúa las funciones cognitivas, Castillo-Parra et al. (2009) encontraron diferencias en niños entre 7 y 8 años (segundo grado de primaria), entre el rendimiento académico alto y bajo, pero no con el medio, y como el área de atención y funciones ejecutivas no diferenció 
entre los tres niveles, concluyeron que la diferencia significativa entre alto/bajo es solo por memoria (la otra área del NEUROPSI). Comparados estos con el grupo de sexto grado, donde sí hubo diferencia significativa entre los tres niveles de rendimiento académico en atención y funciones ejecutivas, concluyeron que en los primeros años de la educación primaria la capacidad de memoria sería más requerida que las funciones ejecutivas.

Aún con las distancias del caso respecto al constructo Funciones ejecutivas en la batería del NEUROPSI utilizada (Guanajuato-Méjico), así como la mayor cuantía en la diversidad de niveles de rendimiento académico y la muestra en general, nos parece pertinente referir sus resultados en relación con los obtenidos por nosotros en IE y PC como funciones cognitivas ejecutivas, y en particular el de PC que examina el grado de madurez neuropsicológico, con la salvedad que de Guanajuato solo se reporta máximo las puntuaciones por área como es la de atención y funciones ejecutivas, donde funciones ejecutivas es una subárea.

Nuestros resultados señalan que del primer al tercer grado de primaria en la ciudad de Lima, IE y PC no difieren significativamente ni por sexo, ni por NRA, ni por GE, pero sí se diferencian por edad operatoria, entre concreto y preconcreto, como resultado de la diferencia de medias de los extremos del rango total de edad en cada muestra.

En la aplicación del NEUROPSI en Guanajuato, "la población estudiada [ $\bar{x}=$ 9.7, $\mathrm{DE}=2.11]$ (...) se clasifica dentro de un rango de ejecución con alteraciones leves a moderadas (70-84 puntos)" (Castillo-Parra et al., 2009: 47). Es decir, las muestras de segundo GE $(\overline{\mathrm{X}}=7.65, \mathrm{DE}=0.479)$ y de sexto $\mathrm{GE}(\overline{\mathrm{x}}=11.74$, $\mathrm{DE}=0.521)$ tuvieron un desempeño cognitivo similar.

Precisamente, nuestros grupos comparables por su promedio de edad son los de segundo grado, $\bar{x}=7.44$ en M1 y $\bar{x}=7.71$ en M2 (Tabla 3). Además de los mayores en primer grado y los menores en tercero grado, los de segundo grado no entraron al contraste por edad operatoria por encontrarse en la interfase de los preconcretos, cuyos promedios de edad fueron $\overline{\mathrm{X}}=6.33$ en M1 y $\overline{\mathrm{X}}=6.50$ en M2 y los concretos, $\overline{\mathrm{x}}=8.83$ en M1 y $\overline{\mathrm{x}}=8.75$ en M2 (Tabla 5).

Al ser mayor la diferencia de edad entre segundo y sexto grado en Guanajuato que en preconcretos y concretos en Lima, se podría esperar que aquellos puntúen diferente en el NEUROPSI, salvo por no contar en su muestra con niños de 6 años.

En relación con el NRA, al haber quedado cortos con el nivel de rendimiento bajo y al contrario con el nivel medio (Tabla 10), nuestros resultados (Tablas 13 y 14) no pueden ser concluyentes al respecto, no sin antes haber correlacionado las calificaciones escolares en las muestras con los totales de las escalas B-O y BTd sin encontrar relación significativa de estos con el total o promedio general del rendimiento escolar, incluso con las áreas de matemática y comunicación integral por separado.

En Atención y funciones ejecutivas en el segundo GE de Guanajuato, al parecer, su relación con las calificaciones escolares no fue significativa tampoco, aunque no ha sido explicitado como sí lo fue en Memoria. 
En Aliaga et al. (2012), tendríamos el caso extremo si relacionamos la inteligencia espacial de las inteligencias múltiples (Gardner) con el pensamiento espacial (Luria, Piaget e Inhelder) al que se remite finalmente la IE y la PC. Aunque en el estudio de las inteligencias múltiples, el test no es De ejecución como los utilizados aquí para representar al pensamiento espacial, reserva que debemos tomar para efectos de la comparación, es pertinente referir los resultados de la correlación de la inteligencia espacial y materias escolares además del total o media de sus calificaciones. Todas fueron correlaciones negativas, en algunos casos, significativos y, en otros, muy significativas. No está de más hacer notar que la inteligencia musical en relación con las calificaciones escolares tuvo un comportamiento similar al espacial, las únicas.

De otro lado (Tabla 15), la correlación de los totales entre IE y PC $(r=0.60, p$ $=0.001)$ permitiría extender por extrapolación lo de las ganancias generacionales con los resultados obtenidos (línea base) de no encontrarse mayor evidencia empírica que permita observar el devenir del pensamiento espacial en los preescolares respecto con la población escolar.

En lo que respecta a la capacidad de las escalas de discriminar genéticamente por edad operatoria, se asume que son instrumentos predictores del desarrollo cognitivo espacial, aspecto importante de evaluar en los niños que ingresan al mundo escolar en nuestro medio donde, al parecer, no se estimula ese desarrollo, sobre todo en los primeros grados.

Por tanto, los resultados de la presente investigación son útiles como punto de referencia en nuestro medio para emprender investigaciones comparativas con otros test de carácter ejecutivo y de desarrollo basados en teorías que se ocupan del comportamiento representativo del espacio, pero necesariamente es preciso confirmar estos resultados en muestras representativas de la población escolar.

\section{CONCLUSIONES}

1) La aplicación de la escala ejecutiva Borelli-Oléron en la población estudiada tiene puntuaciones válidas y confiables en la medición de la inteligencia ejecutiva.

2) La aplicación del test de praxia constructiva tridimensional en la población estudiada tiene puntuaciones válidas y confiables en la medición de la praxia constructiva.

3) La inteligencia ejecutiva no difiere significativamente según grado escolar, sexo y nivel de rendimiento académico en la población estudiada, pero sí difiere según edad operatoria concreta y preconcreta.

4) La praxia constructiva no difiere significativamente según grado escolar, sexo y nivel de rendimiento académico en la población estudiada, pero sí difiere según edad operatoria concreta y preconcreta. 
5) Los resultados prueban la hipótesis de que la praxia constructiva se relaciona positivamente con la inteligencia ejecutiva. Las correlaciones entre las puntuaciones total-total de las escalas fue muy significativa. Asimismo, la mayoría de las correlaciones cruzadas ítem-test total fueron significativas, siendo más elevadas las correlaciones entre los ítems de la escala Benton Tridimensional con el total de la escala Borelli-Oléron.

\section{NOTA DE RECONOCIMIENTO}

Agradecemos al colegio América Callao High School y al colegio Reina de los Ángeles por haber permitido el acceso a la institución para la realización del estudio y, especialmente, a las colaboradoras externas Irene Rispa Z., quien diseñó el procedimiento estadístico, y Jordana Jurado R., por el procesamiento computarizado de la data.

\section{REFERENCIAS}

Alarcón, R. (2013). Métodos y diseños de investigación del comportamiento. (Reimp.). Lima: Universidad Ricardo Palma.

Aliaga, J., Ponce, C., Bulnes, M., Elizalde, R., Montgomery, W., Gutiérrez, V., Delgado, E., Perea, J. y Torchiani, R. (2012). Las inteligencias múltiples: evaluación y relación con el rendimiento en matemática en estudiantes del quinto año de secundaria de Lima Metropolitana. Revista de Investigación en Psicología, 15 (2), 163-202.

Benton, A. (1968). Manuel Test de Praxie Constructive Tri-dimensionelle. [Manual del Test de Praxia Constructiva Tridimensional]. Paris: Centre de Psychologie Appliquée.

Benton, A. L. (2010). Manual del Test de Praxia Constructiva Tridimensional. (Trad. J. C. Rivera B.). Anexo de Informe de Investigación (código 131801101). Manuscrito inédito. Instituto de Investigaciones de la Facultad de Psicología, UNMSM, Lima. (Original en francés, 1968).

Borelli, M. et Oléron, P. (1964). Manuel Echelle de Performance Borelli-Oléron. [Manual de la Escala Ejecutiva Borelli-Oléron]. $\left(2^{\circ}\right.$ Ed.). Paris: Centre de Psychologie Appliquée.

Borelli, M. y Oléron, P. (2000). Manual de la Escala Ejecutiva Borelli-Oléron. En Rivera, J. C. Estudio de la imagen mental reproductora estática y cinética en sujetos con retardo mental leve y moderado del programa de educación especial. (Tesis de Maestría). Recuperado de http://cybertesis.unmsm.edu.pe/ handle/cybertesis/1715 (2012). 
Castillo Parra, G., Gómez, E. y Ostrosky-Solís, F. (2009). "Relación entre las Funciones Cognitivas y el Nivel de Rendimiento Académico en Niños". Revista Neuropsicología. Neuropsiquiatría y Neurociencias, Abril 2009, Vol. 9 , $\mathrm{N}^{\mathrm{o}}$ 1, pp. 41-54. Recuperado de http://neurociencias.udea.edu.co/revista/PDF/ REVNEURO_vo19 num1_8.pdf (2012).

De Montpellier, G. (1973). El Aprendizaje. En Fraisse, P. y Piaget, J. (Comp.). Aprendizaje y Memoria. (Trad. L. Zaltz y M. T. Cevasco). Cap. 2 (pp. 53135). Buenos Aires: Paidós. (Tratado de Psicología Experimental, Vol. 4). (Original en francés, 1968).

Droz, R. y Rahmy, M. (1984). Cómo leer a Piaget. (Trad. A. Romero). México: Fondo de Cultura Económica. (Breviarios 359). (Original en francés, 1978).

Elorza, H. (2008). Estadística para las ciencias sociales del comportamiento y de la salud. ( $3^{\circ}$ Ed.). México, D. F.: CENGAGE Learning.

Garret, H. E. (1974). Estadística en Psicología y Educación. (3 ${ }^{\mathrm{a}}$ Ed.). (Trad. J. Tomas). Buenos Aires: Paidós. (Volumen 22, Serie Mayor). (Original en inglés, $5^{\mathrm{a}} \mathrm{Ed}$.).

Gómez, J. y Giménez, N. (1972). Adaptación de la Escala de Performance de Borelli-Oléron. Resumen de Tesina, presentada por los autores en septiembre, para la obtención del grado de Licenciado de la Universidad de Barcelona, dirigida por el Dr. Miguel Siguán Soler. Recuperado de http://www.raco.cat/ index.php/AnuarioPsicologia/article/viewFile/59749/96204

Hernández, R., Fernández, C. y Baptista, P. (2010). Metodología de la Investigación (5 Ed.). México D.F.: McGraw-Hill.

Kolb, B. y Whishaw, I. Q. (2006). Neuropsicología Humana. (Trad. S. Cwi). Cap. 21 y 23. Madrid: Editorial Médica Panamericana. (Original en inglés).

León, F. (2012). Efectos económicos y psicología de la latitud. Revista de Investigación en Psicología, 15 (2), 217-241.

Luria, A. (1974). El cerebro en acción. (Trad. M. Torres). Barcelona: Fontanella. (Original en ruso).

Luria, A. R. (1977). Las Funciones Corticales Superiores del Hombre (Trad. C. Pérez). La Habana: Orbe. (Original en ruso, 1969).

Matute, E., Rosselli, M., Chamorro, Y. y Orozco, J. (2010). Pruebas neuropsicológicas infantiles en español. En Rosselli, M., Matute, E. y Ardila, A. Neuropsicología del desarrollo infantil. Cap. 5 (pp. 119-136). México: El Manual Moderno.

Piaget, J. (1972). El Nacimiento de la Inteligencia en el Niño. (Trad. L. Fernández C.). Madrid: Aguilar. (Original en francés, 1959). 
Piaget, J. et Inhelder, B. (1972). La représentation de l'espace chez l'enfant. [La representación del espacio en el niño]. ( $2^{\circ}$ Ed.). Paris: Presses Universitaires de France.

Rappaport, D. (1965). Test de diagnóstico psicológico. (Trad. E. Loedel). Argentina: Paidós. (Biblioteca de Psicometría 13). (Original en inglés).

Rivera, J. (2013). Clasificación del dominio cognitivo de las funciones ejecutivas y la memoria en la Batería NEUROPSI. Revista de Investigación en Psicología, $16(1), 145-151$.

Rivera, J. C. (2011). Estudio de la Imagen Mental Reproductora Estática y Cinética en Sujetos con Retardo Mental Leve y Moderado del Programa de Educación Especial. (Tesis de Maestría). Recuperado de http://cybertesis.unmsm.edu.pe/ handle/cybertesis/1715 (2012).

Smith, M. (1975). Estadística simplificada para psicólogos y educadores. (Trad. G. Anguiano e I. Campos). (Reimp.). México: El Manual Moderno. (Original en inglés, 1970). 